A mediados del mes de diciembre de 2009 y marzo de 2010 se realizaron un par de recorridos de exploración en la zona conocida como Ejido San Diego, al suroeste de la cabecera municipal de Río Verde con la finalidad de llevar a cabo una evaluación biológica para el pago de servicios ambientales. A través del acceso a dicho ejido, la vegetación cambia en un gradiente altitudinal que va de los 990 m desde los matorrales xerófilos en las llanuras de Río Verde y bosques tropicales caducifolios al pie de la sierra hasta los 1,872 m s.n.m. en el Cerro La Cruz, donde la vegetación dominante se compone de bosques de encino y de pino. No obstante, en las cañadas húmedas y protegidas de la exposición solar donde se producen escorrentías en época de lluvias, se registraron varias especies arbóreas asociadas con el bosque mesófilo de montaña (en lo sucesivo bmm): Alnus jorullensis Kunth., Carya ovata (Mill.) K.Koch, Cornus disciflora Moc. \& Sessé ex DC., Liquidambar styraciflua L., Quercus germana Schlecht \& Cham. y Q. sartorii Liebm. (Rzedowski, 1961; Puig, 1991; García-Sánchez et al., 1999; ZavalaChávez, 2003; Villaseñor, 2010).

Los bmm registrados se presentan en varias cañadas angostas como fragmentos aislados que se distribuyen en un polígono que abarca 428 hectáreas aproximadamente (Figura 1). La estructura del estrato arbóreo es densa y rebasa los $30 \mathrm{~m}$ de altura donde el elemento Liquidambar se encuentra inmerso entre varias especies de Quercus, lo mismo pasa con Alnus y Carya que se limitan a cañadas con arroyos e igualmente están dispersas entre las especies dominantes de Quercus. Las especies acompañantes del estrato arbóreo fueron: Cupressus lusitanica Mill., Juglans mollis Engelm., Persea sp., Quercus rugosa Née, Q. laeta Liebm., Q. crassifolia Humb. \& Bon-

\title{
NOTA SOBRE UN FRAGMENTO DE BOSQUE MESÓFILO DE MONTAÑA DEL MUNICIPIO de Río Verde, San Luis Potosí
}

\author{
María Magdalena Salinas-RodríGuez ${ }^{1,3}$ Y \\ JuAn Cruzado-Cortés ${ }^{2}$ \\ ${ }^{1}$ Facultad de Ciencias Forestales, Universidad Autónoma de Nuevo León \\ ${ }^{2}$ Calle Abasolo \# 400, Col. Moderna, Linares, Nuevo León, México \\ ${ }^{3}$ Autor para la correspondencia: madreselva_84@hotmail.com
}

pl., Q. obtusata Humb. \& Bonpl., Q. germana Schltdl. \& Cham., Q. affinis Scheidw., Q. sartorii Liebm., Q. potosina Trel. y $Q$. resinosa Liebm.

El estrato arbustivo es más abierto y va de los dos a los siete metros en promedio. Se compone principalmente de elementos jóvenes pertenecientes a las mismas especies que corresponden al estrato arbóreo, no obstante también incluye especies como Arbutus xalapensis Kunth, Cornus disciflora DC., Garrya laurifolia Hartw. ex Benth., Senecio aschenbornianus S. Schauer. y en algunos afloramientos rocosos está presente Brahea decumbens Rzed. Por su parte, el estrato herbáceo es el más variado y en las áreas umbrosas, húmedas o cercanas a los arroyos encontramos: Adiantum capillus-veneris L., Arisaema dracontium (L.) Schott, Aspidotis meifolia (D.C. Eaton) Pic. Serm., Cheilanthes aemula Maxon, Llavea cordifolia Lag., Paspalum notatum Flüggé, Pecluma alfredii (Rosenst.) M.G. Price, Pleopeltis polylepis (Roem. ex Kuntze) T. Moore, Polypodium polypo- dioides (L.) Watt, P. thyssanolepis A. Braun ex Klotzsch, Pteridium caudatum (L.) Maxon, Thelypteris sp. $\mathrm{y}$ Xanthosoma robustum Schott.

Mientras que en las partes con mayor incidencia solar se registraron las siguientes especies herbáceas: Ageratum corymbosum Zuccagni, Anagallis arvensis L., Asclepias curassavica L., Bletia reflexa Lindl., Chamaedorea sp., Croton ciliatoglandulifer Ortega, Eragrostis sp., Hydrocotyle mexicana Cham. \& Schltdl., Lupinus montanus Kunth, Oenothera rosea L'Her. ex Aiton, Oplismenus sp., Oxalis corniculata L., Plantago australis Lam., Salvia coccinea Buc'hoz ex Etl., S. polystachya Cav., Sisyrinchium sp., Veronica persica Poir., Viola flagelliformis Hemsl. y Viola sp.

También se registraron varias especies de hábito rupícola: Agave celsii Hook., Echeveria sp., Pinguicula moranensis Kunth, P. lilacina Schltdl. \& Cham., Sedum greggii Hemsl., Selaginella pallescens (C. Presl) Spring y de hábito epífito: Epidendrum propinquum A. Rich. \& Galeotti, Tillandsia 


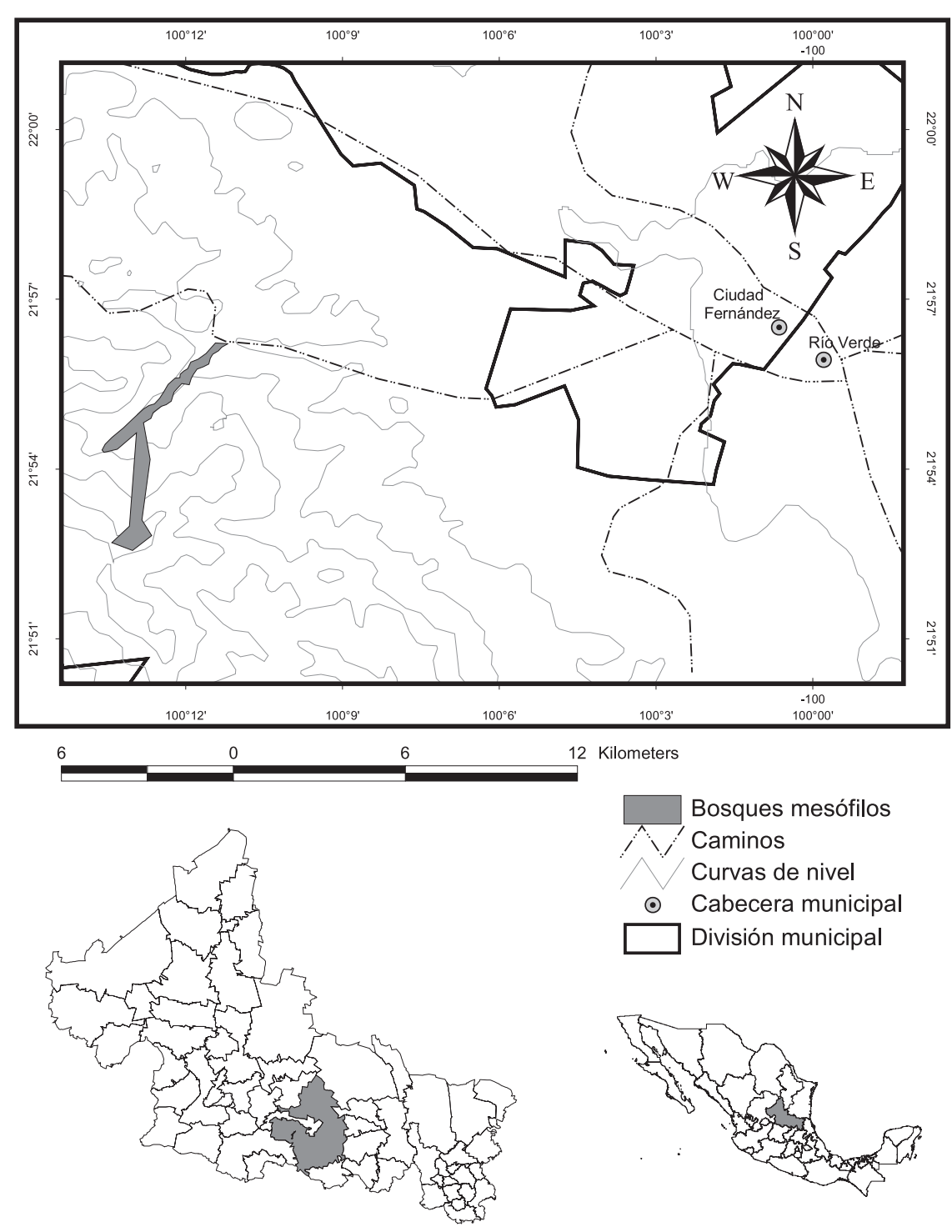

Figura 1. El polígono que se observa en la figura abarca aproximadamente 428 hectáreas, dentro de las cuales se distribuyen varios fragmentos aislados con vegetación de bosque mesófilo de montaña.

recurvata (L.) L. y T. usneoides (L.) L.

Consultando la literatura sobre los bmm regionales (Rzedowski, 1961; Rzedowski, 1978; Puig, 1991; Rzedowski, 1996; García-Sánchez et al., 1999; Cartujano et al., 2002; Sánchez et al., 2003; Valdéz-Tamez et al., 2003; CONABIO, 2010; García-Pérez y Fortanelli-Martínez, 2010; Villaseñor, 2010), encontramos que la CONABIO (2010), hace mención de un conjunto de fragmentos de bmm en los municipios de Ciudad del Maíz, El Naranjo, Alaquines y Tamasopo y los denomina "Vestigios de San Luis Potosí", los cuales se ubican en promedio a $60 \mathrm{~km}$ de distancia hacia el noreste de nuestra área de estudio, los cuales han sido florísticamente estudiados por García-Pérez y FortanelliMartínez (2010) en las localidades de Copalillo, municipio de Tamasopo y La Reforma, municipio de Alaquines y con quienes los bmm de Río Verde comparten especies como Carya ovata, Liquidambar styraciflua y Quercus sartorii, pero no Alnus jorullensis. Debido a que la investigación de GarcíaPérez y Fortanelli-Martínez (2010) no ha sido publicada formalmente en extenso, no fue posible realizar un estudio comparativo completo. Así mismo Villaseñor (2010) menciona que existen fragmentos de bmm en la Sierra de Álvarez en el municipio de Villa de Zaragoza, en promedio a 36 $\mathrm{km}$ de distancia al oeste. Ninguna de estas investigaciones menciona la presencia de bmm en el municipio de Río Verde, por lo que su existencia podría significar una conexión más, entre los relictos de este tipo de vegetación en el estado de San Luis Potosí.

Resulta de utilidad destacar la existencia de estos fragmentos de bmm por su valor fitogeográfico y por proveer servicios ambientales hidrológicos y de biodiversidad a los habitantes de Río Verde. Aunque dentro del camino que pasa por los bosques sólo hay dos rancherías (Montesumas y Paso del Agua), el turismo es nulo y la densidad poblacional del área ha experimentado una reiterada migración de sus pobladores hacia las ciudades o hacia el extranjero, la conservación de estos bosques sigue siendo precaria debido a que los ejidatarios aprovechan la madera y existe presión por parte del ganado vacuno y porcino. Creemos que una exploración más extensa en tiempo y espacio proporcione un panorama completo de los cañones aledaños al ejido y la posibilidad de encontrar nuevas áreas con este tipo de bosque en las sierras del municipio de Río Verde.

\section{Literatura citada}

Cartujano S., Zamudio S., Alcántara O. y Luna I. 2002. El bosque mesófilo de montaña en el municipio de Landa de Matamoros, Querétaro, México. Boletín de la Sociedad Botánica de México 70:13-43.

CONABIO [Comisión Nacional para el 
Conocimiento y Uso de la Biodiversidad]. 2010. El Bosque Mesófilo de Montaña en México: Amenazas y Oportunidades para su Conservación y Manejo Sostenible. Comisión Nacional para el Conocimiento y Uso de la Biodiversidad, México, D.F.

García-Sánchez F., Aguirre-Rivera J.R., Villanueva-Díaz J. y García-Pérez J. 1999. Contribución al conocimiento florístico de la Sierra de Álvarez, San Luis Potosí, México. Polibotánica 10:73-103.

García-Pérez J. y Fortanelli-Martínez J. 2010. Estudio florístico de dos relictos de bosque de niebla de San Luis Potosí. En: Rodríguez-Contreras A., VargasPonce O., Vargas-Amado G, Harker M. y Monroy-Sais A.S. Eds. Memorias del XVIII Congreso Mexicano de Botánica, pp. 350, Sociedad Botánica de
México-Universidad de GuadalajaraUniversidad Autónoma Metropolitana, Guadalajara.

Puig H. 1991. Vegetación de la Huasteca (México). Estudio Fitogeográfico y Ecológico. Institut Francais de Recherche Scientifique pour le Développement en Coopération-Instituto de Ecología-Centre dítudes Mexicaines et Centraméricaines, México, D.F.

Rzedowski J. 1961. Vegetación del Estado de San Luis Potosí. Tesis doctoral, Facultad de Ciencias, Universidad Nacional Autónoma de México, México, D.F. 228 pp.

Rzedowski J. 1978. Vegetación de México. Limusa, México, D.F.

Rzedowski J. 1996. Análisis preliminar de la flora vascular de los bosques mesófilos de montaña de México. Acta Botanica Mexicana 35:25-44.
Sánchez O., Vega E., Peters E. y MonroyVilchis O. Eds. 2003. Conservación de Ecosistemas Templados de Montaña en México. Instituto Nacional de Ecología, México, D.F.

Valdéz-Tamez V., Foroughbakhch-Pournavab R. y Alanís-Flores G. 2003. Distribución relictual del bosque mesófilo de montaña en el noreste de México. Ciencia UANL 4:360-365.

Villaseñor J.L. 2010. El Bosque Húmedo de Montaña en México y sus Plantas Vasculares: Catálogo Florístico-Taxonómico. Universidad Nacional Autónoma de México-Comisión Nacional para el Conocimiento y Uso de la Biodiversidad, México, D.F.

Zavala-Chávez, F. 2003. Identificación de Encinos de México. Universidad Autónoma de Chapingo, Chapingo.

Recibido: 29 de junio de 2011

Aceptado : 24 de agosto de 2011 